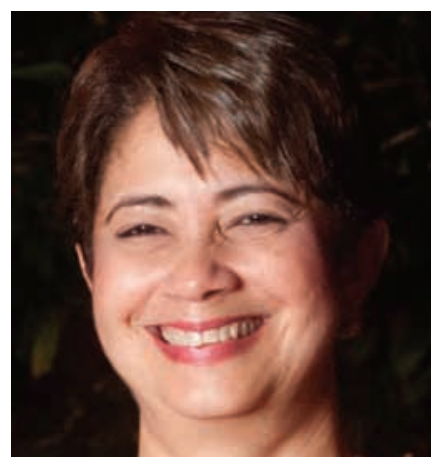

\title{
Valoración de Implicaciones para la Formación en Residencia Médica de Anestesiología
}

\section{Por Evangelina Hernández *}

Resumen: Se presenta una sistematización de los procesos de enseñanza-aprendizaje de la residencia médica de Anestesiología en uno de los hospitales universitarios avalada por la Pontificia Universidad Católica Madre y Maestra. Se analizaron documentos relacionados a la educación superior en postgrado, se realizaron entrevistas semiestructuradas a los implicados en el proceso de formación. Se finaliza el trabajo interpretando los resultados como una fuente de información y de argumentación para debatir en cuanto a la enseñanza-aprendizaje de las residencias médicas, tomando como caso la de anestesiología. Se hace énfasis en la importancia de aplicar los reglamentos del nivel de postgrado y afianzar los aspectos pedagógicos, para el logro de los objetivos concretos de un profesional competente de la salud en esta área.

Abstract: This article reports on a systematic organization of the teaching and learning process in the Anesthesiology medical residency certified by the Pontificia Universidad Católica Madre y Maestra at a university hospital. Documents related to Higher Education at the graduate level were analyzed and semi-structured interviews to the medical residents were conducted. The results highlight the importance to apply the regulations at the graduate level as well as the need to enhance the pedagogical aspects, in order to achieve the specific objectives of a proficient health professional. Finally, it is expected that these results could be used as a source of information and argumentation for future debate over the teaching and learning process at medical residencies.

\section{Construcción de la pregunta}

Como profesora docente de la residencia en Anestesiología en la Pontificia Universidad Católica Madre y Maestra (PUCMM), nace mi inquietud sobre los procesos de enseñanza y aprendizaje utilizados por los docentes del área en el Hospital Regional Universitario Presidente Estrella Ureña (HRUPEU).

La Sociedad Americana de Anestesiología (ASA) define la Anestesiología como: "la práctica de la Medicina dedicada al alivio del dolor y al cuidado completo e integral del paciente quirúrgico, antes, durante y después de la cirugía". [sitio web] www. anestesia.com.mx. Un anestesiólogo es el doctor encargado del paciente a todo lo largo del procedimiento quirúrgico. Esto incluye suprimir el dolor, vigilar los signos vitales durante la cirugía y la con- tinuación de los cuidados postoperatorios hasta que el paciente esté en condiciones estables para ser llevado a planta, manteniendo una estrecha comunicación con el equipo quirúrgico. Lo que podría incluir situaciones de emergencia, manejo de ciertos cuadros clínicos y una participación activa en el quirófano y fuera del hospital.

Los médicos residentes son profesionales egresados de diferentes universidades. En el caso de Anestesiología, existen 3 plazas para optar: los dos primeros lugares pertenecen al Instituto del Seguro Social (IDSS) y un tercero a la Secretaría de Estado de Salud Pública y Asistencia Social (SESPAS). Ambas instituciones se encargan de pagar al residente durante los años que dure su formación. La permanencia en la especialización se gestiona a través de un contrato renovable cada

\begin{abstract}
* Médico Anestesiólogo y Especialista en Pedagogía Universitaria por la Pontificia Universidad Católica Madre y Maestra. Profesora por asignatura en la Facultad de Ciencias de la Salud de esta Universidad. Asesora de Proyectos Finales Residencias Médicas en Anestesiología, avaladas por la PUCMM. Coordinadora por seis años de la residencia anestesiología Hospital Regional Universitario Presidente Estrella Ureña (IDSS). Para contactar a la autora: evangelina.hg@gmail.com
\end{abstract}

Encuentre el texto en "La Investigación sobre la Práctica Docente", http://www.pucmm.edu.do/STI/campus/CDP/ComuncacionPublicaciones/Paginas/CuadernodePedagogiaUniversitaria.aspx

Cuaderno de Pedagogía Universitaria Año 9/ N. 17 /enero-junio 2012 / Santiago, Rep. Dom.: PUCMM / p. 38-43
Recibido el 15-Mayo-12 Aprobado el 12-Oct-12 
año mientras cumpla con los requisitos del reglamento. Iniciándose así a un programa de formación y de entrenamientos específicos de cuatro años, durante los cuales el residente, de acuerdo a su grado, debe de adquirir los conocimientos teóricos y prácticos implicados en el plan de estudios de la Residencia en Anestesiología de la PUCMM. A cada nivel se le asigna el nombre de Residente I, II, III ó IV, de acuerdo al año que cursa (Plan de Estudios de la Residencia en Anestesiología vigente desde el 2002. PUCMM).

El programa de la especialidad tiene como propósito el desarrollo de competencias para la formación y el perfeccionamiento de recursos humanos en el ámbito de una ocupación, profesión, disciplina, arte o técnica, según el Reglamento del Nivel de Postgrado de las Instituciones de Educación Superior, Ministerio de Estado de Educación Superior, Ciencia y Tecnología (2008).

Las residencias médicas están dirigidas por tres niveles de mando y la universidad. La PUCMM actúa a través del Director de la Cátedra de la Especialidad, de la Facultad de Ciencias de la Salud, que avala la residencia. Labor que se realiza conjuntamente con el IDSS, a través del Director General de Enseñanza e Investigaciones Médicas y con el Colegio Médico Dominicano, a través del Presidente de la Sociedad Especializada, relativa a la residencia médica, en este caso la de Anestesiología. Los niveles de mando y la PUCMM se rigen por las leyes del Ministerio de Educación Superior, Ciencia y Tecnología, la Ley General de Salud, la Ley de Organización del Cuerpo Médico de los Hospitales y el Consejo Nacional de Residencias Médicas. Sus funciones son establecer el programa, evaluar su desarrollo y dotar de recursos humanos y bibliográficos para la formación.

El ambiente de formación en la Residencia Médica constituye un compromiso didáctico: debe existir un personal docente que aplique estrategias de enseñanza-aprendizaje para el desarrollo del pensamiento analítico y crítico y retroalimentación entre los docentes y el residente en forma- ción. Las complejidades del proceso no se han sistematizado, de manera que puedan conocerse las implicaciones del mismo. "La base para que una educación en anestesia sea eficaz debe basarse en una comprensión del pasado y un análisis del presente, pero, lo más importante, debe ser determinada por nuestra visión para el futuro" (Longnec 2002).

A partir de lo expuesto, mi inquietud para emprender esta investigación fue la siguiente: ¿Cómo se valora y cómo se ha desarrollado la formación en la Residencia Médica de Anestesiología?

\section{Recopilación de la evidencia}

Esta investigación constituye una sistematización de experiencias en la docencia de Anestesiología en el HRUPEU. Ruiz (2001), expresa que la sistematización es "un proceso de recuperación, tematización y apropiación de una práctica formativa determinada, que al relacionar sistémica e históricamente sus componentes teórico-prácticos, permite a los sujetos comprender y explicar los contextos, sentido, fundamentos, lógicas y aspectos problemáticos que presenta la experiencia, con el fin de transformar y cualificar la comprensión, experimentación y expresión de las propuestas educativas de carácter comunitario."

Se recogieron datos documentales y se realizaron entrevistas semiestructuradas. Los informantes fueron: a) el decano de postgrado, b) la encargada de las residencias médicas de la PUCMM, c) el jefe de docencia médica del hospital, d) el coordinador de la residencia en el hospital, e) los profesores docentes de la residencia en Anestesiología de la PUCMM, f) los residentes del hospital de los cuatro años de residencia, g) los documentos y registros de postgrado. Las entrevistas fueron personales, vía electrónica o telefónica durante tres meses.

En las tablas 1, 2 y 3 se desglosan y se describen los criterios a partir de los cuales la información fue recopilada y analizada, acorde a las intenciones más específicas (objetivos), formuladas a partir de la pregunta principal.

Tabla1. Criterios e indicadores de análisis para el objetivo 1

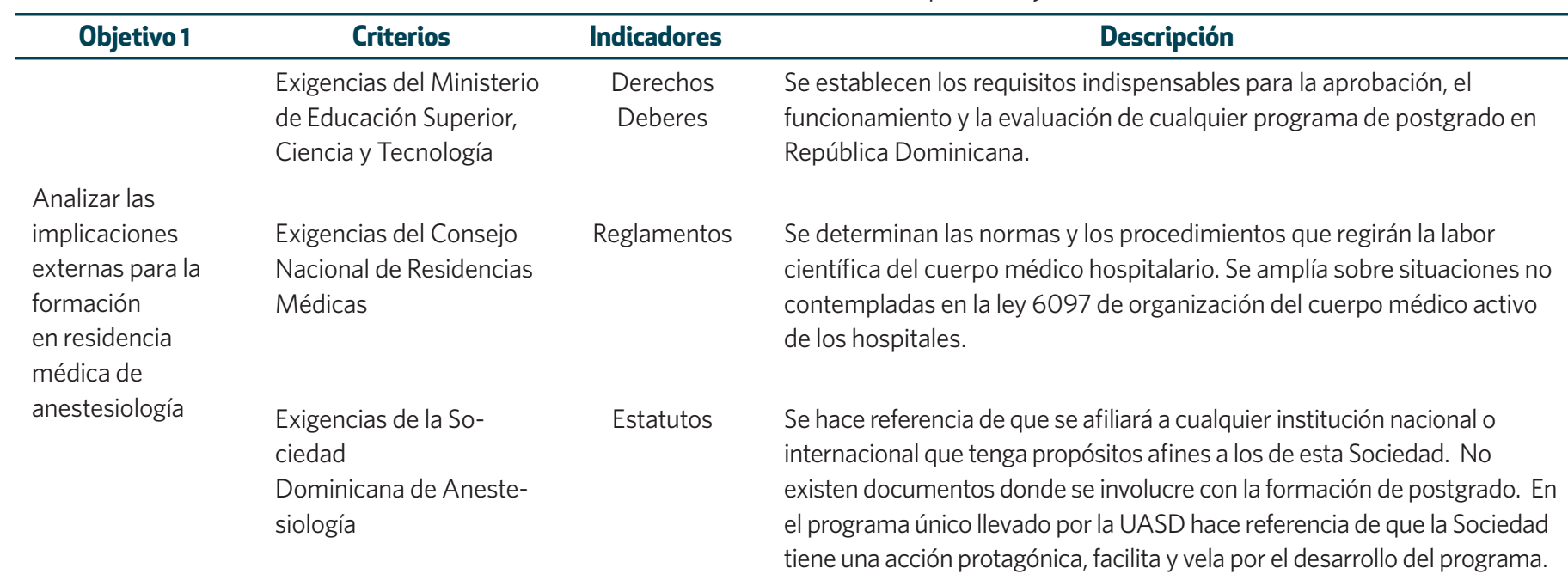

Valoración de Implicaciones para la Formación en Residencia Médica de Anestesiología: Sistematización de Experiencias, Evangelina Hernández Cuaderno de Pedagogía Universitaria Año 9/ N. 17 /enero - junio 2012 / Santiago, Rep. Dom.: PUCMM / p. 38-43 
Tabla 2. Criterios e indicadores de análisis para el objetivo 2

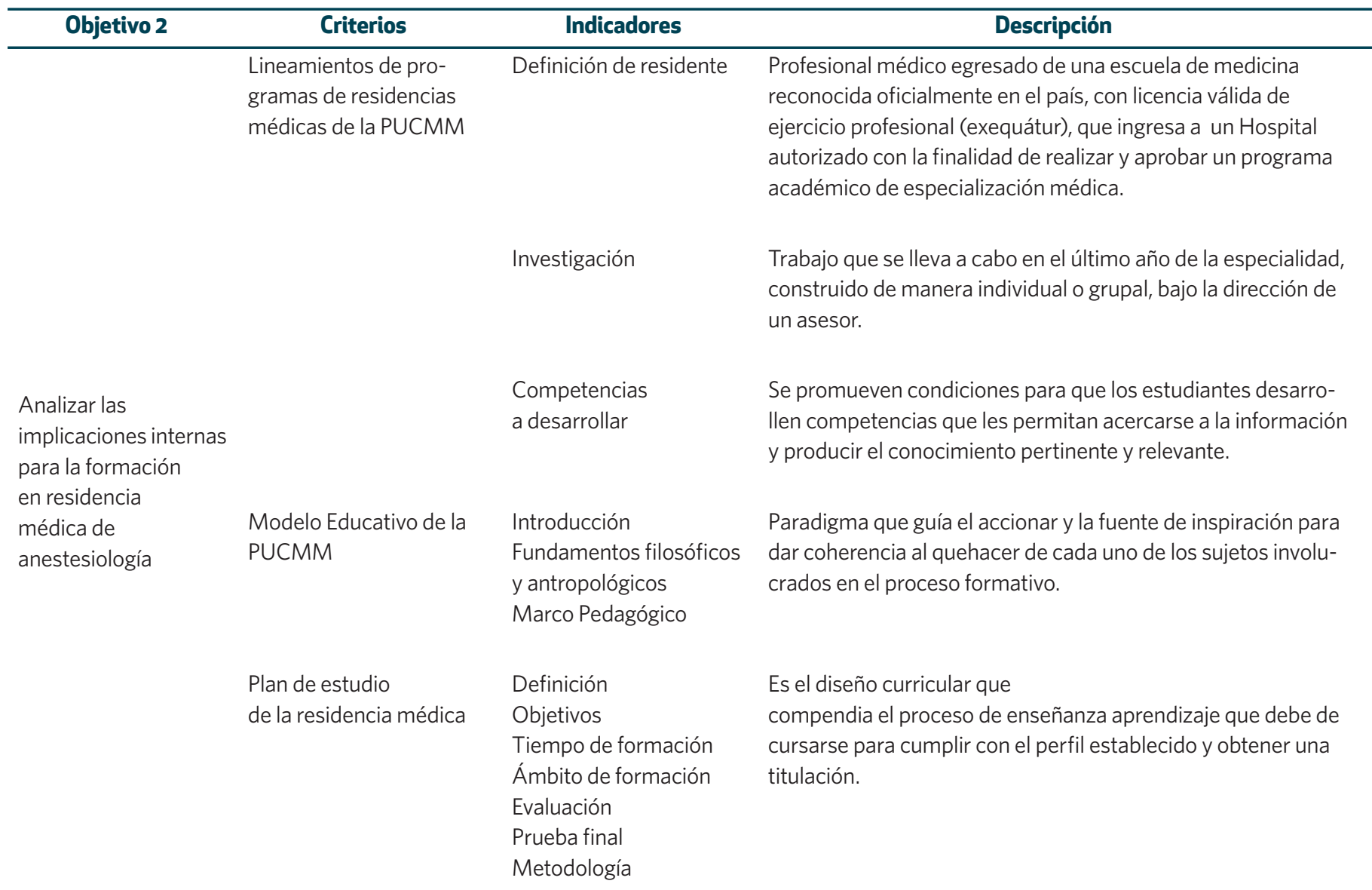

Valoración de Implicaciones para la Formación en Residencia Médica de Anestesiología: Sistematización de Experiencias, Evangelina Hernández Cuaderno de Pedagogía Universitaria Año 9/ N. 17 /enero - junio 2012 / Santiago, Rep. Dom.: PUCMM / p. 38-43 
Tabla 3. Criterios e indicadores de análisis para el objetivo 3

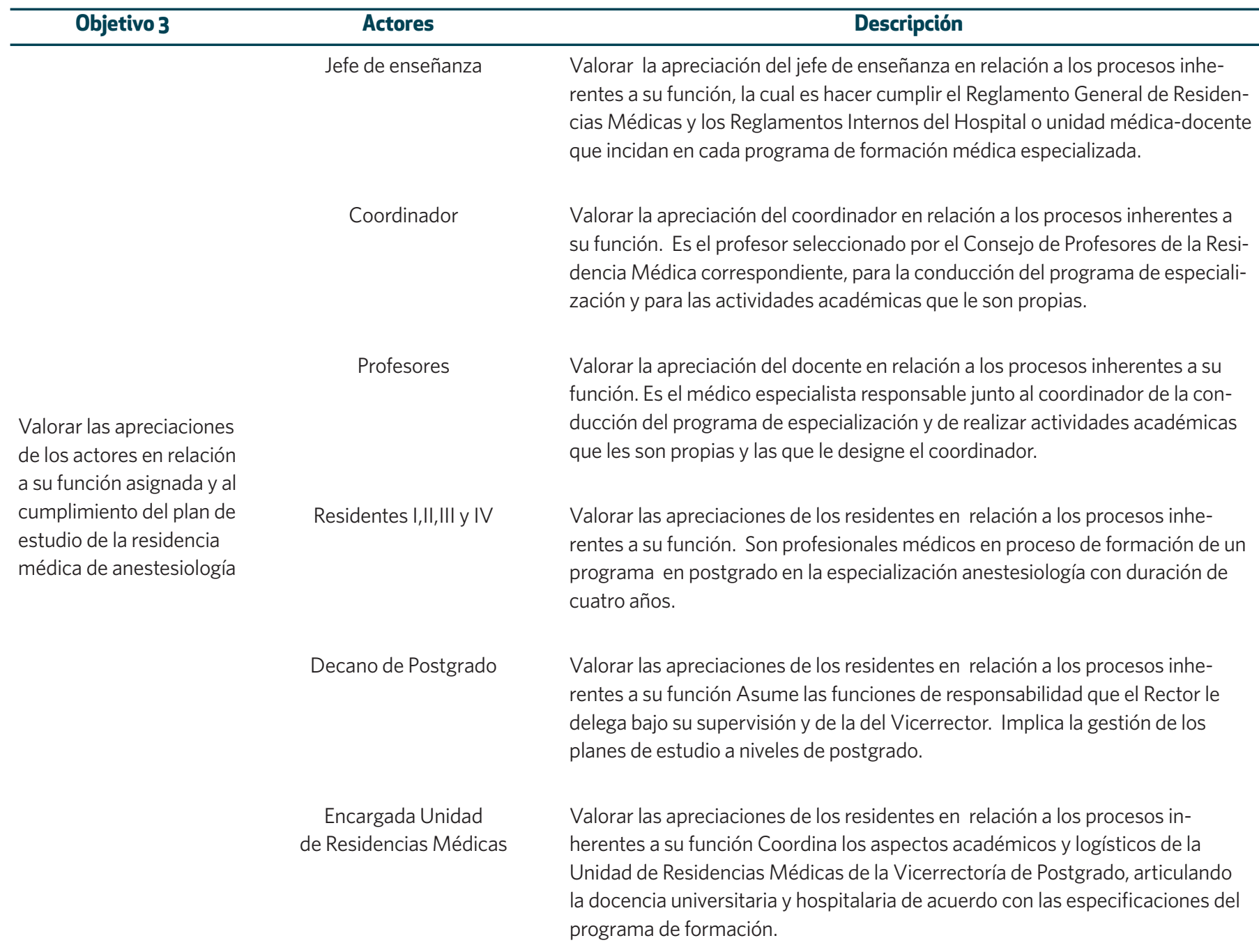

Para el cumplimiento de estos objetivos se utilizaron como fundamentación referentes disciplinares, pedagógicos y metodológicos; a saber, plan de estudios de la Residencia Médica, Competencias implicadas en los programas de postgrado en Anestesiología, Proyecto Tuning, Declaración de Bolonia, los estándares en Educación Médica de Postgrado del Proyecto de la World Federal for Medical Education (WFME), Organización de las Naciones Unidas para la Educación, la Ciencia y la Cultura (UNESCO), Reglamento del Nivel De Postgrado de las Instituciones de Educación Superior del Ministerio de Estado de Educación Superior, Ciencia y Tecnología (MEESCYT), Ley 139-01 de Educación Superior, Ciencia y Tecnología, Ley General de Salud. No. 42-01, Ley de Organización del Cuerpo Médico de los Hospitales (Número 6097), Reglamento Nacional de Residencias Médicas del Consejo Nacional de Residencias Médicas en la República Dominicana (Reglamento Único), Lineamientos de Postgrado, Modelo Educativo, Plan de Estudio y sobre las implicaciones de la sistematización.
Luego de la lectura, análisis y valoración de los diferentes documentos relacionados a las implicaciones externas e internas de la residencia en Anestesiología del HRUPEU y de las apreciaciones de los actores involucrados en la misma presento las conclusiones a continuación.

En cuanto a las implicaciones externas se concluye que en el documento referido a las exigencias del Ministerio de Educación Superior, Ciencia y Tecnología, la Institución, a nivel de Postgrado, debe incentivar la superación académica y la actualización periódica de sus profesores. Se registran iniciativas que evidencian el esfuerzo de la PUCMM y la motivación por la superación académica docente a través de diversas ofertas de formación, tanto a niveles disciplinares, como pedagógicos.

La revisión del documento de las exigencias del Consejo Nacional de Residencias Médicas, permite apreciar que son muchos los acápites que deben ser reevaluado, en todas las instancias involucradas en la 
formación de especialistas para el mejor desenvolvimiento futuro de las residencias médicas, en todos los niveles. Es justificable la necesidad de reestructurar y evaluar por parte de las autoridades estos reglamentos, donde se haga referencia de las propiedades facultativas y académicas de la PUCMM, que le da la autoridad de participación en un nivel I, lo que les permitirá estar a la par con el proceso de reforma del sector salud.

Las exigencias de la Sociedad Dominicana de Anestesiología reúnen un conjunto de regulaciones de carácter moral, que codifican la práctica honesta y conducta honorable de todos y cada uno de los médicos anestesiólogos afiliados a cualquiera de las Sociedades Miembros de Confederación Latinoamericana Anestesiología, del médico ya especializado. No existe contenido en sus documentos donde se involucre con la formación de postgrado, ni de lo que espera del especialista en formación. Solamente el Programa Único llevado por la UASD hace referencia de que la Sociedad tiene una acción protagónica, que facilita y que vela por el desarrollo del programa.

En relación con las implicaciones internas, los lineamientos de programas de residencias médicas de la PUCMM, indican antecedentes, la motivación y la justificación de su diseño y elaboración. En el mismo se dan nociones sobre formación y desarrollo de la investigación en los programas de postgrado, se dan directrices para la construcción de líneas y temáticas para un trabajo de investigación, la evaluación y corrección, que son necesarios para que un trabajo a este nivel tenga buena aceptación y credibilidad. Por ello, a nivel de postgrado existen unos criterios, normativas y estándares de evaluación en la investigación, que garantizan su cumplimiento. Como usuaria del documento testifico que ofrecen orientación; sin embargo, algunas implicaciones no están contempladas.

El Modelo Educativo de la Institución constituye una guía como fuente de inspiración para dar coherencia al quehacer de los sujetos involucrados en el proceso formativo. Se evidencia que la Universidad persigue un desarrollo metodológico participativo, de construcción de conocimientos, desarrollo de destrezas, habilidades, valores y actitudes, necesarios para desenvolverse en una sociedad competitiva y convivir en ella haciendo trascender sus acciones, dando sentido a la vida y lograr un futuro profesional competente, mediante procesos de acompañamiento y tutorías.

El plan de estudio de la residencia médica, documento creado en un principio para orientar a todo personal de salud en el área formativa de residencia médicas, en especial en Anestesiología, hace énfasis en los objetivos y las actividades programadas por año en la residencia. Cada nivel tiene sus actividades específicas en el aspecto cognoscitivo, las habilidades que se quiere que se adquieran para lograr un profesional competente. El documento incluye estrategias que persiguen la supervisión; sin embargo, ha sido hasta el momento irregular, por el no comprometimiento de todos los especialistas o docentes. Se está reevaluando y reestructurando, ya que fue emitido hace ya muchos años y debe ser actualizado, principalmente en lo que respecta a las especificaciones correspondientes al cuarto año, el cual fue adicionado luego de su elaboración.
En otro orden, las apreciaciones de los actores sobre las implicaciones indican diversos aspectos importantes resaltar. Coinciden en la concepción de la especialidad, sus conceptos objetivos y también coinciden en considerar que es importante que se verifiquen constantemente el cumplimiento de los mismos. En cuanto al tiempo de formación ven la necesidad de que roten por otros hospitales, tanto del país como del extranjero, que aprendan de diversas prácticas docentes. Su opinión acerca del ámbito de formación es que se beneficiaría más con la participación activa de todos los especialistas del Departamento.

Con respecto a la evaluación, las opiniones difieren, unos responden que en cierto sentido está incompleta; en cambio, otros dicen que es adecuada y llena los parámetros establecidos. En lo que concierne a la prueba final expresan que reúne los requisitos para saber o evaluar al residente. Una problemática identificada radica en el hecho de que los especialistas no van a las entregas de guardia, los que realmente deben evaluar esos aprendizajes. Para finalizar, en relación a la metodología de enseñanza-aprendizaje opinan que debería existir un seguimiento formalizado, más estricto y que la residencia se beneficiaría de un mayor apoyo por parte de los especialistas.

\section{Conclusiones}

No ha existido un seguimiento permanente relacionado con el mecanismo de evaluación, acreditación y desarrollo de la residencia de Anestesiología de la PUCMM en el HRUPEU. No existen mecanismos de evaluación, monitoreo y seguimiento para el cumplimiento de las funciones de los actores involucrados, ni el cumplimiento económico para el profesional docente. Esta situación no ayuda a incentivar al docente en profundizar en su trabajo, pues lo percibe como una función no remunerada. Los docentes, por tanto, dejan a los residentes solos más tiempo de lo conveniente. La actividad del residente suele centrarse en el aspecto laboral, lo cual le consume gran parte del período que debía estar dedicado al estudio, hasta impedir su asistencia habitual al recinto universitario donde se le imparten temas teóricos que son imprescindibles en su formación.

Los entrevistados en esta investigación informaron que existe falta de interés desde el hospital con respecto a la formación de los residentes. Los residentes, en particular, hicieron énfasis en que tienen la necesidad de que el especialista docente se involucre más con ellos. Esperan que se tome conciencia de un aprendizaje más significativo y desean que se valore la calidad del entorno en que se da la formación. En ocasiones, estas condicionantes les hacen pensar que su educación como especialistas no está completa.

Las competencias a desarrollar en los residentes deben ser abordadas por una educación continua que permita la actualización y perfeccionamiento; donde se impliquen diversas modalidades: cursos, seguimiento por parte de los docentes, elaboración de protocolos y diversas estrategias didácticas.

La formación profesional en las residencias médicas requiere la coordinación e integración curricular y la preparación pedagógica de los docentes. Una formación que incluya aspectos relevantes de las ciencias 
biomédicas, básicas, clínicas, sociales, al igual que de habilidades de comunicación, ética médica; una disciplina necesaria en la que se demuestre una práctica profesional en el área, un aprendizaje integrado donde se puedan evaluar conocimientos, actitudes y aptitudes.

Existe la necesidad de que se evalúen y reestructuren los reglamentos de Consejo Nacional de las Residencias Médicas, los cuales datan de hace 40 años. Se requiere que la Universidad cuente con más poder de decisión para formar a los especialistas, de manera que pueda involucrarse más y asumir un proceso de crecimiento. La sistematización de experiencias en las otras residencias médicas de Anestesiología avaladas por la PUCMM favorecería la conformación de un plan único de formación.

En los aspectos pedagógicos, es pertinente identificar los cambios en lo que respecta a la práctica pedagógica al concluir cada ciclo o año de residencia. Esto así, para lograr integrar mejores estrategias y medios tecnológicos apropiados para cada nivel. Como en una de sus exigencias del Ministerio de Educación Superior, Ciencia y Tecnología, (2008), expresa que la política institucional a nivel de Postgrado debe incentivar la superación académica y la actualización periódica de sus profesores.

El proceso de enseñanza-aprendizaje debe enfocarse en un aprendizaje secuencial, con retroalimentación permanente durante el proceso y posibilidad de refuerzos. Es preciso incluir procesos metacognitivos a través de los cuales el residente tome conciencia del desarrollo de sus habilidades. El Consejo General de Residencias Médicas, el Hospital Universitario y la Universidad deben actuar en coordinación para el asesoramiento y supervisión y se pueda mejorar la calidad de la formación, para lograr capturar los significados de todas sus acciones y sus efectos, con tal de no repetir esos errores que se han llevado a cabo, mejorar las propias prácticas, compartir los aprendizajes. Un proceso de asesoramiento, supervisión y comunicación para que se realicen líneas de acción y se pueda construir más aportes en la formación de postgrado en medicina de Anestesiología.

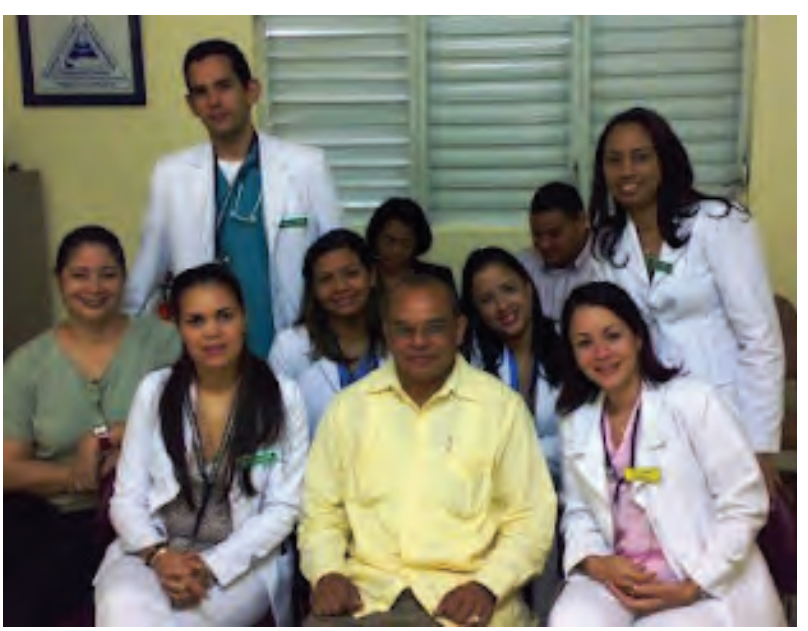

Es preciso un proceso de reconstrucción y reflexión analítica, que sea participativo, desde el Consejo General de Residencias Médicas, PUCMM y el Hospital Universitario con los docentes y residentes, para lograr capturar los significados de todas las acciones y sus efectos.

Para lograr cambios y construir el futuro, debemos analizar el pasado y el presente. En los años que estuve en la coordinación de la residencia médica no tuve la oportunidad de conocer la existencia de los reglamentos y las exigencias de postgrado. Este hecho me impidió comprender muchos pormenores y llegué a caer en las mismas incongruencias que muchos en la actualidad mantienen. Esta falta de conocimiento ha llevado a adjudicarle a la Universidad las fallas internas en las residencias, puesto que es la Institución que avala la formación. Sin embargo, esta creencia es un error. La Universidad no tiene el control del proceso en su totalidad, ya que las autoridades gubernamentales del área de salud son las que determinan la apertura de los puestos de residencia y la elaboración del plan de estudio, sin contemplar la efectividad sobre el contexto real.

Se necesita más empoderamiento por parte de la Universidad, para lograr mejoría en el desenvolvimiento de la formación de nuevos especialistas, para tener un poder de decisión, tener acceso a la información y a los recursos, para tomar decisiones apropiadas, mejorar la imagen e involucrarse en un proceso de crecimiento. Las residencias médicas constituyen la base de la educación médica de postgrado y debe contar con la infraestructura, la organización, los medios y el personal necesario para que asuma la responsabilidad de un plan de estudio, además del apoyo de la Sociedad Dominicana de Anestesiología (SDA), para mantener los objetivos fijados y los estándares determinados actualizados, además de que defina los indicadores que permitan su evaluación y acreditación.

Reitero la necesidad de renovar las oportunidades de formación y actualización para promover, perfeccionar y desarrollar las aptitudes docentes para incorporar saberes epistemológicos, pedagógicos y metodológicos. El perfeccionamiento docente podría programarse como un proceso de formación permanente para la revisión y renovación de conocimientos, la revisión de actitudes y habilidades, un perfeccionamiento docente que ayudará a analizar la calidad de las estrategias de aprendizaje que tradicionalmente se realizan en el hospital.

\section{Referencias Bibliográficas}

Ruiz, B. \& Dary, L. (2001). Sistematización de Prácticas. Foro Los contextos actuales de la educación popular. 20 de septiembre de 2001. Medellín: Liceo Nacional Marco Fidel Suárez.

Longnec, D (2002). Education in anesthesiology for the twenty-first century. Journal Anesthesiology 16, 65-69.

Ministerio de Estado de Educación Superior, Ciencia y Tecnología, (MEESCyT). Reglamento del Nivel de Postgrado de las Instituciones de Educación Superior. Santo Domingo: MEESCyT 